\title{
Etude ethnobotanique et phytochimique des plantes médicinales utilisées dans le traitement des maladies cardiovasculaires à Moundou (Tchad)
}

\author{
Delphine NGUEMO DONGOCK ${ }^{1 *}$, Alexandre LAOHUDUMAYE BONYO ${ }^{1,2}$, \\ Pierre Marie MAPONGMESTEM ${ }^{1}$ et Elysée BAYEGONE ${ }^{2}$
}

\author{
${ }^{1}$ Laboratoire de Biodiversité et de Développement Durable, Département des Sciences Biologiques ; \\ Faculté des Sciences; Université de Ngaoundéré, Cameroun. \\ ${ }^{2}$ Université de N'Djamena, Tchad. \\ *Auteur correspondant, E-mail: dndongock@yahoo.fr; \\ BP : 454 Ngaoundéré, Cameroun ; Tel. (237) 699451096.
}

\section{RESUME}

Les maladies cardiovasculaires sont responsables de nombreux problèmes de santé dans le monde. En effet, ces maladies constituent un ensemble de troubles qui touchent le cœur et la circulation sanguine. Parmi ces maladies, les plus récurrentes sont le diabète et l'hypertension artérielle. L'objectif principal de ce travail est de contribuer à la connaissance des plantes médicinales utilisées dans le traitement du diabète et de l'hypertension artérielle au Tchad, aussi d'effectuer le screening phytochimique de quelques espèces sollicitées. Une enquête ethnobotanique s'est déroulée dans 216 ménages à Moundou dans le Logone occidental. Au total, 58 espèces dont 55 genres et 27 familles ont été recensées, parmi lesquelles, 19 espèces soignent le diabète, 17 l'hypertension artérielle et 22 sont utilisées pour les deux maladies. Fabaceae Caesalpinoideae, Combretaceae et Euphorbiaceae sont les familles les plus représentées. Toutes les parties de la plante sont sollicitées. Néanmoins les feuilles $(27,32 \%)$, l'écorce du tronc $(16,82 \%)$ et les racines $(14,76 \%)$ sont les parties les plus sollicitées. La décoction (62\%), la macération $(23,66 \%)$ et l'infusion $(14,34 \%)$ sont les modes de préparation les plus fréquents. Les recettes sont très variées en fonction des groupes ethniques. L'analyse phytochimique de Sclerocarya birrea, Annona senegalensis, Detarium microcarpum, Cassia sieberiana, Momordica charantia et Phyllantus amarus indique leur richesse en tanins, flavonoïdes, alcaloïdes et saponosides. Ces composes sont des éléments indicateurs qui justifieraient leur utilisation dans le traitement des maladies cardiovasculaires.

(C) 2018 International Formulae Group. All rights reserved.

Mots clés: Phytochimie, plantes médicinales, diabète, hypertension artérielle, Tchad.

\section{Ethnobotanic and phytochemical study of medicinal plants used for the treatment of cardiovascular diseases in Moundou (Chad)}

\begin{abstract}
Cardiovascular diseases constitute a major health problem in the world today. In fact, these diseases are pathologies that affect the heart and blood circulation. Among these diseases, the most recurrent are diabetics and hypertension. The main objective of this study was to contribute to the better knowledge of medicinal plants used for the treatment of diabetes and hypertension in Chad, also to carry out the phytochemical
\end{abstract}


screening of some species requested. An ethnobotanic survey was carried out in 216 household in Moundou, West Logone. A total of 58 species, 55 genera and 27 families were identified among which 19 species treat diabetes, 17 hypertension and 22 used in combination to treat the two diseases. Fabaceae, Caesalpinoideae, Combretaceae and Euphorbiaceae were the highest represented families. All parts of plants are used. However, the leaves $(27.32 \%)$, the barks of the trunk $(16.82 \%)$ and the roots $(14.76 \%)$ were parts that are highly needed. The decoction (62\%), the maceration $(23.66 \%)$ and the infusion $(14.34 \%)$ were the most frequent methods of preparation. Phytochemical analysis of Sclerocarya birrea, Annona senegalensis, Detarium microcarpum, Cassia sieberiana, Momordica charantia and Phyllantus amarus indicated their richness in tannins, flavonoids, alkaloids and saponosides. These compounds are indicators elements justifying their usefulness in the treatment of cardiovascular diseases.

(C) 2018 International Formulae Group. All rights reserved.

Keywords: Medicinal plants, phytochemical, diabetic, hypertension, Chad.

\section{INTRODUCTION}

L'efficacité de la phytothérapie est prouvée et ses bienfaits incontestables pour la santé a permis à la médecine naturelle d'entrer dans nos habitudes quotidiennes (Bene et al., 2016). De nos jours, le recours à la médecine par les plantes connaît un regain d'intérêt dans les pays occidentaux, particulièrement pour traiter les déséquilibres entraînés par la vie moderne, qu'il s'agisse du stress ou des problèmes de poids (Adomou et al., 2012; Bene et al., 2016). Cependant, malgré les efforts consentis dans ce domaine, les maladies cardiovasculaires continuent à causer la mortalité humaine à travers le monde. Plus de $80 \%$ de décès dus aux maladies cardio-vasculaires interviennent dans les pays en développement (OMS, 2011; Sharma et al., 2012). L'hypertension artérielle est l'une des maladies cardiovasculaires qui touchent actuellement $26,4 \%$ de la population mondiale et pourraient atteindre $29,2 \%$, soit plus de 1,5 milliards d'individus en 2025 (Ueli et al., 2008). La prévalence de l'hypertension artérielle en Afrique est très importante avec $28 \%$ de la population qui souffre en Afrique subsaharienne et $76 \%$ de ces cas sont liés à l'hypertension artérielle. Au Cameroun, on estime à $25 \%$ soit 5000000 personnes touchées par l'hypertension artérielle (Sharma et al., 2012). Le diabète a un taux de prévalence mondiale estimé à 2,8\% en 2000 avec des projections à $4,8 \%$ en 2030 (Ueli et al., 2008 ; Sakine et al., 2012). Le nombre total de personnes atteintes passerait de 171 millions en 2000 à 366 millions en
2030 si rien n'est fait (Ueli et al., 2008). Au Cameroun, on compte 900000 personnes touchées par le diabète (Idriss, 2012). L'Atlas du diabète (2007) rapporte que le Diabète sera en 2030, la principale cause de mortalité en Afrique subsaharienne (Idriss, 2012). En 2012, Afriquinfo rapporte que la prévalence de cette maladie au Tchad est estimée entre 700.000 et 800.000 personnes, a ces chiffres s'ajoute le manque de spécialistes dans le domaine, l'éloignement, la rareté ou l'inexistence de centres de santé dans les villages, la non disponibilité et le coût trop élevé des produits pharmaceutiques. La population ne pouvant pas faire face à ces maladies a recours à la médecine traditionnelle (Proteus, 2000).

La valorisation de la médecine traditionnelle présente ainsi un intérêt croissant. Selon l'OMS (2011), près de $80 \%$ des populations dépendent de la médecine traditionnelle. Des avantages économiques considérables dans le développement de la médecine traditionnelle et dans l'utilisation des plantes médicinales pour le traitement de diverses maladies ont été constatés (Adjanahoun et al., 1980). Malgré que les plantes médicinales rendent d'énormes services à la population tchadienne, très peu de recherches y ont été consacrées. L'objectif de ce travail est de contribuer à la connaissance des plantes médicinales utilisées dans le traitement des maladies cardiovasculaires au Tchad en vue de mettre au point, dans l'avenir des médicaments traditionnels améliorés. 


\section{MATERIEL ET METHODES}

\section{Site d'étude}

Moundou est situé au sud dans la zone soudano-guinéenne de la République du Tchad, précisément entre $16^{\circ} 05^{\prime} 00^{\prime}$, longitude Est et $8^{\circ} 34^{\prime} 00^{\prime \prime}$ latitude Nord. Moundou est la capitale économique du Tchad. Elle couvre une superficie de $22 \mathrm{~km}^{2}$ avec 152849 habitants (RGPH2, 2009). Le climat est de type Soudano-guinéen avec une pluviométrie annuelle variant entre 922 à $1415,9 \mathrm{~mm}$ de pluies. La saison des pluies va d'avril à octobre avec une humidité relative comprise entre 26 à $98 \%$. Après cette saison de pluies, suit une saison sèche qui va de novembre à mars. Les températures à cette période sont de 19,7 à $34,9{ }^{\circ} \mathrm{C}$ (Sougnabé, 2010). Les sols sont ferralitiques et ferrugineux arrosés par le Logone long de $1000 \mathrm{~km}$, qui prend sa source dans les plateaux de l'Adamaoua au Cameroun (Sougnabé, 2010). La végétation est caractérisée par des forêts claires, et de vaste surface herbeuse formant d'excellents pâturages clairsemés d'arbres parmi lesquelles Vitellaria paradoxa, Parkia biglobosa, Khaya senegalensis, Tamarindus indica, Adansonia digitata.... La population très homogène est constituée essentiellement des Ngambaye, Laka et Kaba. Seules les activités commerciales ont attiré quelques Haoussa, Bornouans ou Arabes. La principale activité économique de cette population est l'agriculture suivie par l'élevage et le commerce. Il existe également d'autres petites activités économiques comme la pêche, la cueillette (Sougnabé, 2010).

\section{Enquête ethnobotanique}

L'enquête ethnobotanique s'est déroulée dans les ménages permettant d'apprécier le savoir endogène des paysans et des tradithérapeutes sur la connaissance des plantes médicinales, leur mode d'emploi et les maladies traitées. A cet effet, deux approches ont été entreprises: la médecine populaire et la médecine spécialisée.

La médecine populaire a utilisé la méthode de Tearfund et al. (2001). Elle a consisté à interviewer des personnes qui ont acquis des connaissances sur l'utilisation des plantes médicinales dès leur enfance pour soigner les malades cardiovasculaires avec succès. Un total de 200 personnes ont été enquêtées soit 50 par groupe ethnique. Les principaux groupes ethniques ciblés ont été les Ngambaye, Laka, Kaba et Arabes.

Concernant la médecine spécialisée, l'approche méthodologique a consisté à repérer des guérisseurs traditionnels ayant reçus des expériences personnelles ou des connaissances transmises depuis environ cinq générations, 16 guérisseurs au total ont été ainsi enquêtés.

Pour chacune de ces méthodes, les interviews individuelles se sont réalisées à l'aide d'un questionnaire préalablement élaboré comportant des questions fermées, ouvertes et orientées. Les grandes lignes du questionnaire relatif à la médecine populaire ont porté sur la perception traditionnelle des maladies cardiovasculaires, les symptômes, les principales plantes utilisées dans le traitement de chacune des maladies, la posologie, les recettes. Pour la médecine spécialisée, les rubriques spécifiques ont abordées l'expérience professionnelle, les symptômes de chacune des maladies, les principales plantes utilisées, la durée du traitement, le mode de préparation du médicament et la disponibilité des plantes. Les échantillons de plantes ont été récoltés et identifiés au Laboratoire de Biodiversité et de Développement Durable de l'Université de Ngaoundéré.

\section{Analyse phytochimique des plantes médicinales}

Six plantes médicinales choisies au hasard utilisées dans le traitement des maladies cardiovasculaires ont été soumises aux analyses phytochimiques. Sur la base des molécules chimiques qui entrent dans les médicaments conventionnels utilisés dans le traitement des maladies cardio-vasculaires, les principaux polyphénols ayant fait l'objet d'une caractérisation chimique ont été les tanins, saponosides, flavonoïdes et alcaloïdes. Les échantillons des plantes ont été récoltés, séchés à l'abri du soleil pendant deux 
semaines, écrasés à l'aide d'un mortier traditionnel puis soumis au screening phytochimique afin de déterminer les grands groupes phénoliques et chimiques: Tanins, Flavonoïdes, Alcaloïdes et les Saponosides (George, 1999).

\section{Analyse statistiques}

Les données quantitatives collectées ont fait l'objet d'une analyse de variance à partir du logiciel Statgraphic plus version 5.0. Le test de Turkey a été utilisé pour séparer les moyennes.

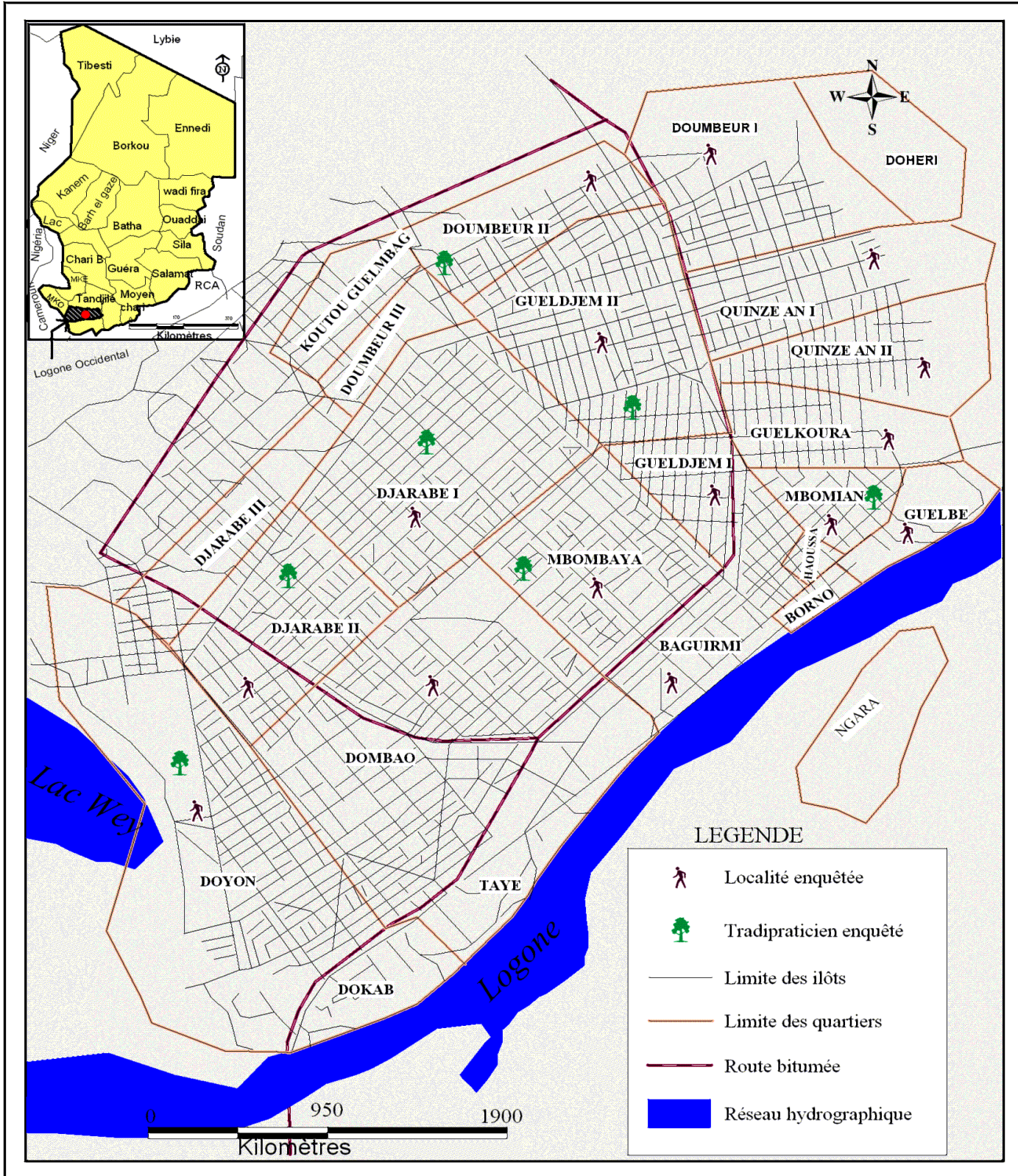

Source:CNAR 2012\& Travaux de terrain 2012

Réproduction et Adaptation Laohudumaye B. Alexandre 2012.

Figure 1: Carte de localisation du site d'étude. 


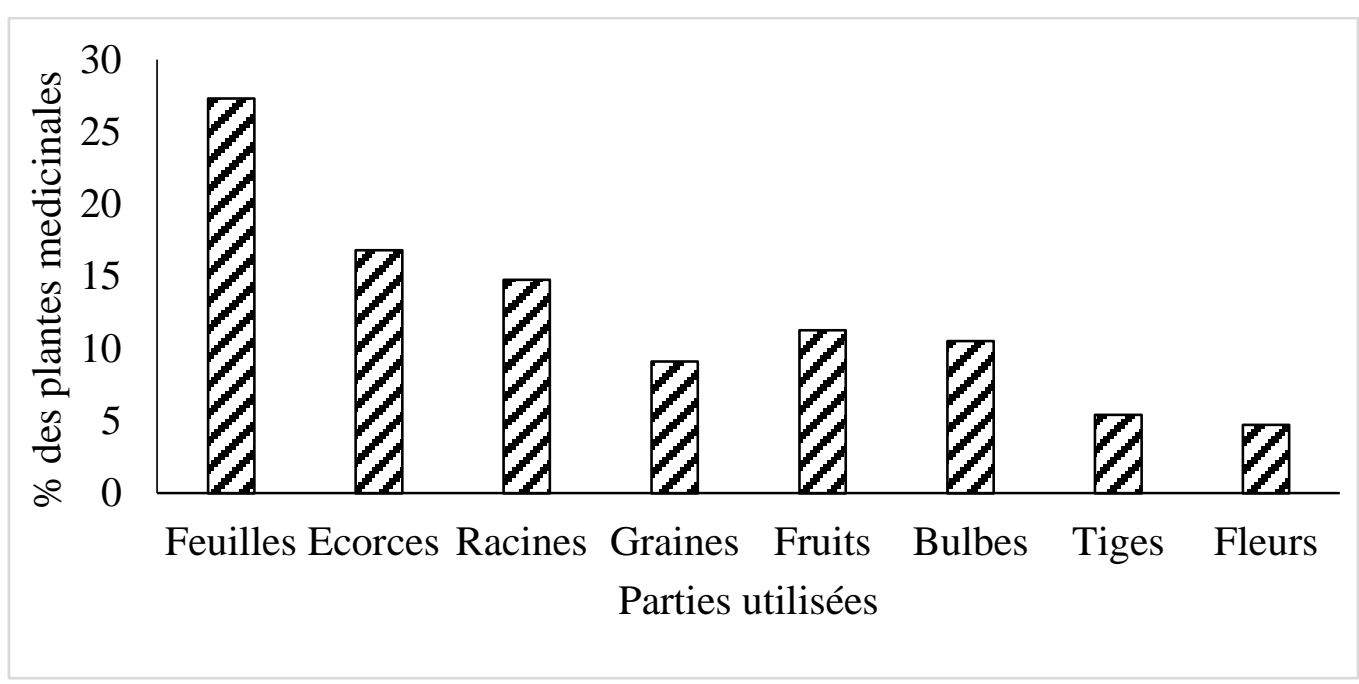

Figure 2: Parties utilisées dans le traitement des maladies cardio-vasculaires.

\section{RESULTATS}

\section{Etat de connaissance des plantes} médicinales

Le savoir endogène sur les plantes médicinales varie d'un groupe ethnique à un autre. Les Ngambaye ont cité 44 et 39 plantes qui soignent respectivement le diabète et l'hypertension, 42 et 37 (Laka), 42 et 37 (Kaba), 12 et 14 (Arabes). Il ressort des enquêtes ethnobotaniques que les populations du Logone occidental utilisent 58 espèces de plantes médicinales reparties en 27 familles et 55 genres pour le traitement du diabète et de l'hypertension artérielle (Tableau 1). La proportion des plantes qui rentrent dans le traitement du couple diabète - hypertension est élevée avec $43,10 \%$, suivie de celles du diabète uniquement $(32,75 \%)$ et de l'hypertension artérielle (24,13\%). Carica papaya, Momordica charantia, Zea mays, Moringa oleifera, Combretum nigricans et Terminalia avicennioides sont fréquemment utilisées dans la préparation des médicaments par toutes les ethnies pour le traitement des deux maladies. Sorghum bicolor (24,75\%), Pithecellobium dulce (23,48\%), Senna siamea $(23,31 \%)$, Annona senegalensis $(22,49 \%)$ et Mangifera indica $(22,12 \%)$ sont fortement sollicitées pour le traitement de l'hypertension artérielle. Quatre espèces sont très sollicitées pour soigner le diabète : Sclerocarya birrea
(82,71\%), Leptadenia hastata (24,52\%), Combretum micranthum (25,55\%), Bauhinia rufescens $(25,10 \%)$. Les familles les plus fréquentes varient en fonction des maladies: diabète (Ceasalpiniaceae, Euphorbiaceae, Liliaceae, et Combretaceae); hypertension artérielle (Apocynaceae, Anacardiacea, Ceasalpiniaceae et Poaceae). Par contre deux familles sont à la fois utilisées dans le traitement des deux maladies: Ceasalpiniaceae et Combretaceae.

\section{Parties des plantes médicinales utilisées}

Toutes les parties des plantes sont utilisées (Tableau 1). Les feuilles $(27,32 \%)$, l'écorce du tronc $(16,82 \%)$ et les racines $(14,76 \%)$ sont les parties les plus utilisées au Logone occidental. L'analyse de variance montre une différence hautement significative $(0,000<0,001)$ entre les parties de plantes. Le groupe ethnique des Arabes n'utilisent pas les graines et les tiges dans la préparation de leur médicament. La grande sollicitation des feuilles dans la médecine traditionnelle, trouverait sa raison dans leurs richesses en substances actives. Les parties utilisées varient également en fonction des espèces et des maladies à soigner, pour le traitement du diabète: Cassia sieberiana, Azadirachta indica, Bauhinia rufescens, Leptadenia hastata, les parties les plus utilisées sont les 
racines, écorces, graines et feuilles. Dans le traitement de l'hypertension artérielle, les racines, écorces et feuilles de Detarium microcarpum, Persea americana, Annona senegalensis, Euphorbia hirta sont couramment utilisées. Les feuilles et écorces, feuilles ou graines, racines ou graines de Sclerocarya birrea, Momordica charantia et Moringa oleifera rentrent dans le traitement des deux maladies.

\section{Modes de préparations des plantes}

Globalement, trois modes de préparation ont été enregistrés (Tableau 1): la décoction $(62 \%)$, la macération $(23,66 \%)$ et l'infusion $(14,34 \%)$. L'analyse de variance montre une différence très significative $(0,0035<0,01)$ entre les trois modes de préparation des médicaments. L'infusion ne rentre pas dans les habitudes de préparation des médicaments chez les Arabes. Au niveau des ethnies, cette différence ne se remarque pas $(1,0000>0,05)$. Les échanges culturels entre ces ethnies peuvent être l'un des facteurs ayant favorisé la vulgarisation des différents modes de préparation dans la localité (Tableau $1)$.

\section{Caractérisation phytochimique des extraits}

Toutes les six plantes soumises au test Phytochimique sont riches en tanins, flavonoïdes, alcaloïdes et saponosides. Seuls les flavonoïdes présentent des précipités peu abondants chez toutes les espèces étudiées comparés aux tanins, flavonoides et alcaloïdes qui présentent des précipités très abondants. L'étude phytochimique de la poudre de l'écorce de Sclerocarya birrea a relevé la présence des tanins, flavonoïdes, alcaloïdes et des saponosides. Le test de caractérisation phytochimique des feuilles de Phyllantus amarus a révélé la présence des tanins, flavonoïdes, alcaloïdes et des saponosides.

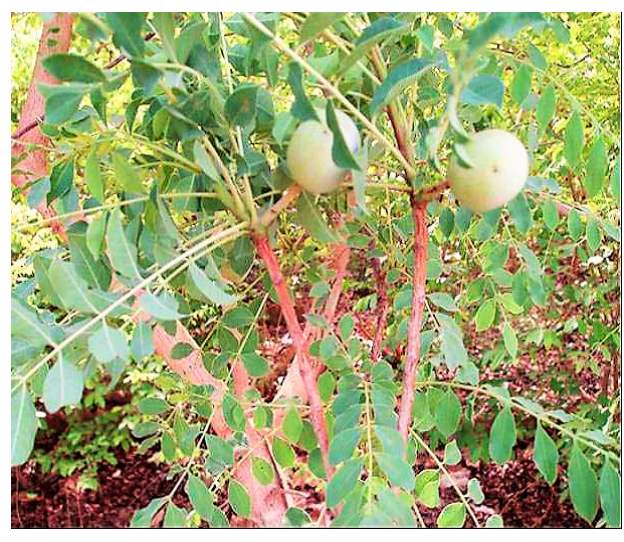

Sclerocarya birrea (A. Rich.) Hochst.

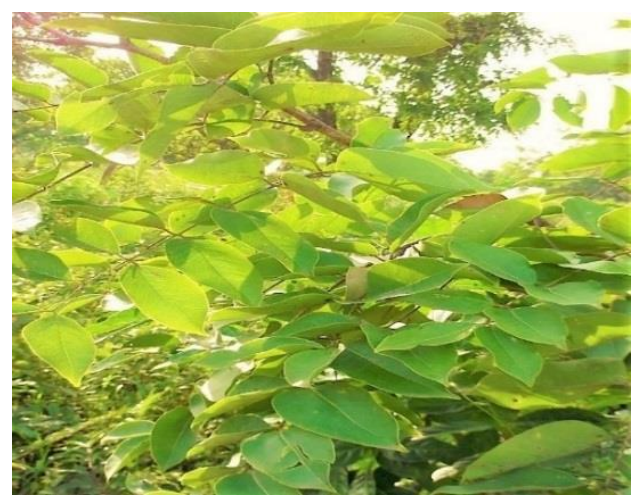

Daniellia oliveri (Rolfe) Hutch. et Dalz

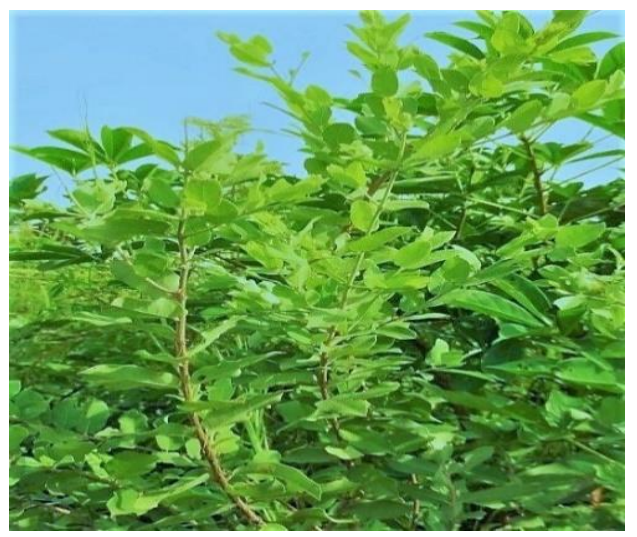

Guiera senegalensis J.F. Gmel.

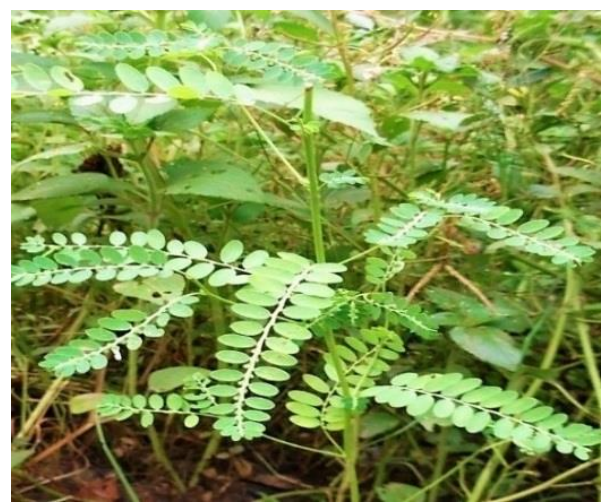

Phyllantus amarus Shumach. 


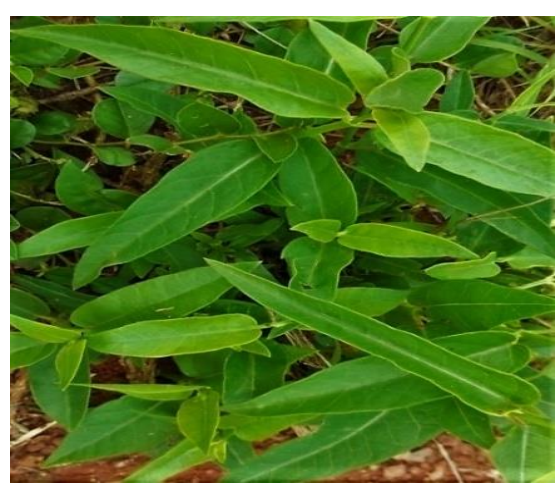

Leptadenia hastata (Pers.) Decne.

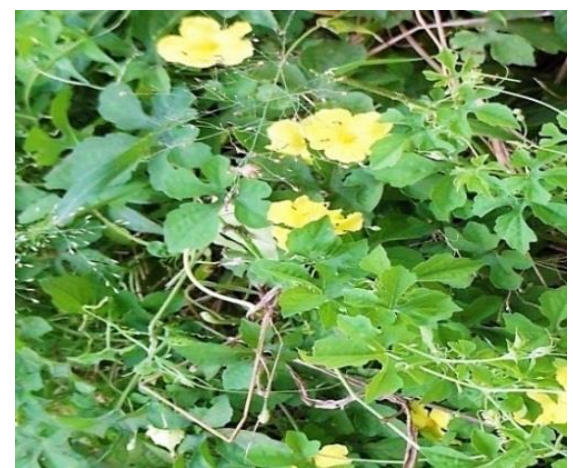

Momordica charantia $L$.

Figure 3 : Photos de quelques espèces végétales utilisées pour le traitement du diabète.

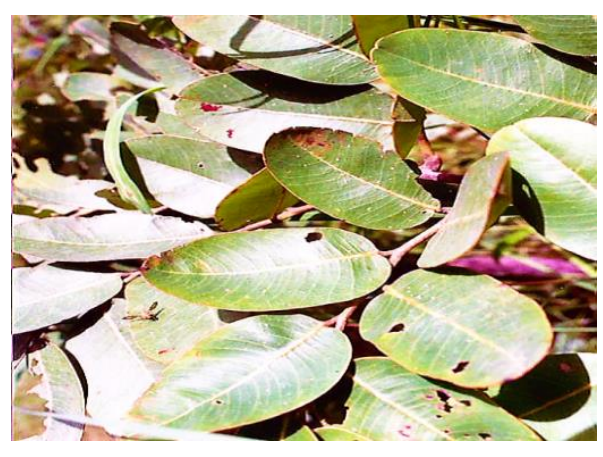

Detarium microcarpum Guill. \& Perr.

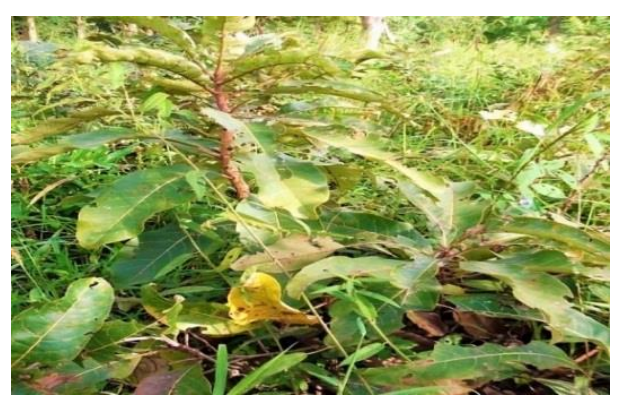

Terminalia avicennioides Guill.et Perr.

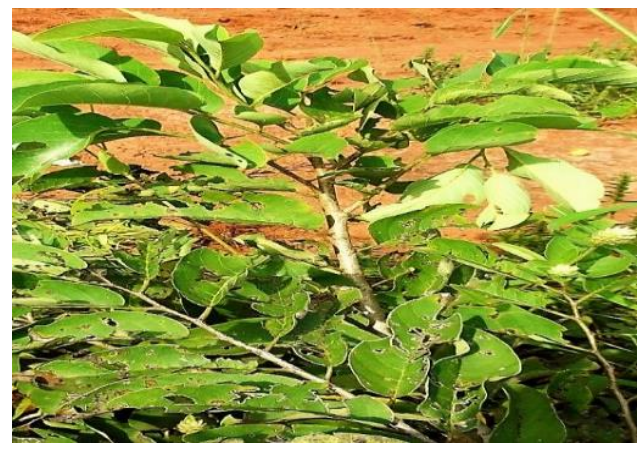

Annona senegalensis Pers.

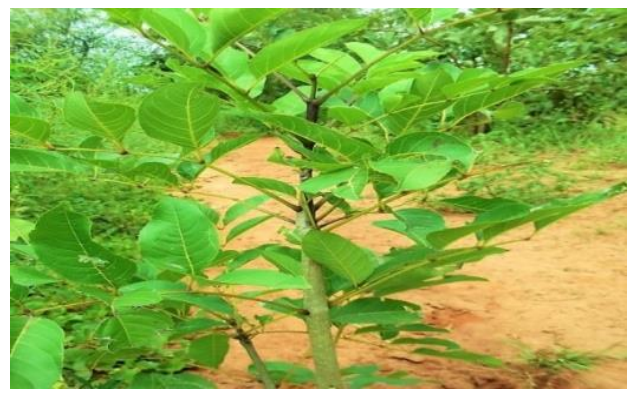

Stereospermum kunthianum Cham.

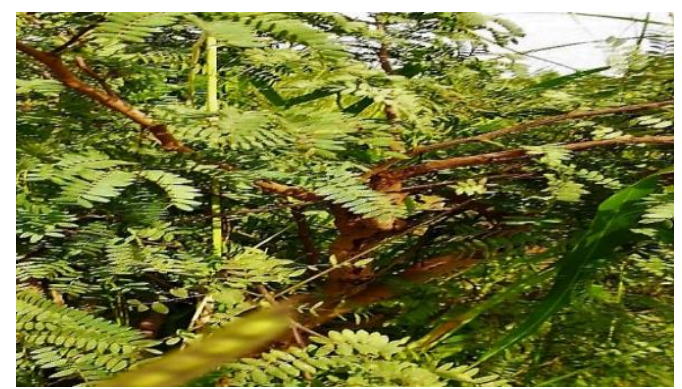

Prosopis africana (Guill. et Perr.) Taub.

Figure. 4: Photos de quelques espèces végétales utilisées pour le traitement de l'hypertension artérielle. 
Tableau 1: Liste des espèces par groupe ethnique, parties utilisées, maladies soignées et posologie.

\begin{tabular}{|c|c|c|c|c|c|}
\hline \multirow[t]{2}{*}{ Noms Scientifiques } & \multirow[t]{2}{*}{ Familles } & \multirow{2}{*}{$\begin{array}{l}\text { Parties } \\
\text { utilisées }\end{array}$} & \multicolumn{2}{|c|}{ Maladies } & \multirow[t]{2}{*}{ Préparation et Posologie } \\
\hline & & & DIA & HTA & \\
\hline Adansonia digitata $\mathrm{L}$. & Bombacaceae & $\mathrm{Fe}$ ou Ec & 11,28 & 6,65 & Décoction, 3 fois/jr pendant une semaine. \\
\hline Afzelia africana Smith ex Pers. & Caesalpiniaceae & Ec & 3,78 & 10,59 & Macération, 1 verre 2 fois/jr pendant 3 jours. \\
\hline Allium сера $\mathrm{L}$. & Liliaceae & Gs & 12,28 & - & Jus, petit verre trois fois/jr pendant une semaine. \\
\hline Allium sativum $\mathrm{L}$. & Liliaceae & Gs & 16,91 & - & Jus, petit verre trois fois/jr pendant une semaine. \\
\hline $\begin{array}{l}\text { Amblygonocarpus andongensis (Welw. } \\
\text { Ex Oliv.) Exell \& Torre }\end{array}$ & Fabaceae & $\mathrm{Fe}$ & 7,08 & 3,04 & Décoction, 3 fois/jr pendant une semaine. \\
\hline Anacardium occidentale L. & Anacardiaceae & $\mathrm{Fe}$ ou Ec & 14,05 & 19,42 & Décoction 3 fois/jr pendant trois jours. \\
\hline Annona senegalensis Pers. & Annonaceae & Rac & - & 22,49 & Décoction, un verre une fois/jr pendant 3 jours. \\
\hline Feretia apodanthera Dell. & Rubiaceae & Rac & - & 5,69 & Décoction, 3 fois/jr Pendant deux jours. \\
\hline Azadirachta indica A. Juss. & Meliaceae & $\mathrm{Gr}$ & 7,69 & - & Macération, petit verre 1 fois/jr pendant 3jours. \\
\hline Balanites aegyptiaca (L.) Del. & Balanitaceae & $\mathrm{Fr}$ & 6,95 & 9,19 & Macération, tous les jours pendant 2 semaines. \\
\hline Bauhinia rufescens Lam. & Caesalpiniaceae & $\mathrm{Fe}$ & 25,10 & - & Décoction, tous les jours pendant une semaine. \\
\hline Bridelia scleroneura Mül. Arg. & Euphorbiaceae & Rac & 10,67 & - & Décoction, tous les jours pendant trois jours. \\
\hline Burkea africana Hook. F. & Caesalpiniaceae & $\mathrm{Fe}$ & 8,43 & 4,92 & Décoction, 3 fois/jr pendant une semaine. \\
\hline Calotropis procera (Aiton) W.T.Aiton. & Apocynaceae & Fr & - & 11,25 & Bain, 2 fois/jr pendant 3 jours. \\
\hline Carica papaya $L$. & Caricaceae & $\mathrm{Fe}$ & 23,66 & 15,80 & Décoction, toute une journée pendant 2 jours. \\
\hline Cassia sieberiana $D C$. & Cesalpiniaceeae & Rac ou Ec & 6,95 & - & Décoction, 1 verre trois fois/jr pendant une semaine. \\
\hline Catharantus roseus (L.) G. Don. & Apocynaceae & $\mathrm{Fe}$ & - & 9,41 & Décoction, 2 fois/jr pendant 3 jours. \\
\hline Combretum glutinosum Perr. & Combretaceae & $\mathrm{Fe}$ & 5,30 & 6,67 & Décoction, 3 fois/jr pendant une semaine. \\
\hline Combretum micranthum G. Don. & Combretaceae & $\mathrm{Fr}$ & 22,55 & - & Décoction, voie orale, 3 fois/jr \\
\hline Combretum nigricans Lepr. ex Guill. & Combretaceae & $\mathrm{Fe}$ & 13,55 & 5,98 & Décoction, 3 fois/jr pendant une semaine. \\
\hline
\end{tabular}


D. NGUEMO DONGOCK et al. / Int. J. Biol. Chem. Sci. 12(1): 203-216, 2018

\begin{tabular}{|c|c|c|c|c|c|}
\hline Daniellia oliveri (Rolfe) Hutch. \& Dalz. & Caesalpiniaceae & Ec & 6,08 & - & Décoction, 2 fois/jr pendant une semaine. \\
\hline Detarium microcarpum Guill. \& Perr. & Caesalpiniaceae & Ec ou Rac & - & 3,20 & Macération, 1verre Une fois/jr, une seule fois. \\
\hline Entada africana Guill.et Perr. & Mimosaceae & Ec & 17,84 & 9,49 & Décoction, 4 à 5 fois/jr pendant 2 jours. \\
\hline Euphorbia hirta L. & Euphorbiaceae & $\mathrm{Pl}$ & - & 15,51 & Décoction, 1 verre trois fois/jr Une seule fois. \\
\hline Ficus polita Vahl. & Moraceae & $\mathrm{Fe}$ & 4,00 & - & Décoction, 3 fois/jr pendant 3 jours. \\
\hline Ficus spp & Moraceae & $\mathrm{Fe}$ & 8,42 & 9,05 & Décoction, tous les jours pendant 3 jours. \\
\hline Grewia cissoides Hutch. & Tiliaceae & $\mathrm{Fe}$ & 5,21 & 5,37 & Décoction, 3 fois/jr pendant une semaine. \\
\hline Grewia mollis Juss. & Tiliaceae & $\mathrm{Fe}$ & 3,54 & 6,26 & Décoction 3 fois/jr pendant une semaine. \\
\hline Guiera senegalensis J.F. Gmel. & Combretaceae & Rac & 15,98 & - & Décoction, 1 verre 2 fois/jr pendant trois mois. \\
\hline Hexalobus monopetalus (A. Rich.) & Annonaceae & $\mathrm{Fe}$ & 4,90 & 3,04 & Décoction, 3 fois/jr pendant une semaine. \\
\hline Hygrofila auriculata (Schum.) & Acanthaceae & $\mathrm{Gr}$ & 6,55 & - & Décoction, tous les jours pendant 1 semaine. \\
\hline Hymenocardia acida Tul. & Phyllanthaceae & $\mathrm{Fe}$ & 4,71 & - & Décoction, 3 fois/jr 1s. \\
\hline Indigofera garckeana Vatke. & Fabaceae & Rac & 9,53 & - & Décoction, 3 fois/jr pendant une semaine. \\
\hline Ipomea batatas (L.) Lam. & Convolvulaceae & $\mathrm{Fe}$ & 14,00 & - & Décoction, 3 fois/jr pendant une semaine. \\
\hline Leptadenia hastata (Pers.) Decne. & Asclepiadaceae & $\mathrm{Fe}$ & 24,52 & - & Macération, 1 verre 3 fois/jr pendant une semaine \\
\hline Mangifera indica $\mathrm{L}$. & Anacardiaceae & $\mathrm{Fe}$ & - & 22,12 & Décoction, 1 verre trois fois/jr une seule fois. \\
\hline Momordica charantia L. & Cucurbitaceae & Fe ou Gr. & 26,61 & 20,07 & Macération, 1 verre trois fois/jr pendant 1 semaine. \\
\hline Moringa oleifera Lam. & Moringaceae & Rac ou Gr. & 14,99 & 15,75 & Avaler 12 graines reparties en 6 jours. \\
\hline Oxytenanthera abyssinica A. Rich. & Poaceae & $\mathrm{Fe}$ & - & 7,52 & Décoction, 3 fois/jr pendant deux jours. \\
\hline Parinari curatellifolia Benth. & Chrysobalanaceae & $\mathrm{Fe}$ & 6,63 & 3,43 & Décoction, 3 fois/jr pendant une semaine. \\
\hline Persea americana Mill. & Lauraceae & $\mathrm{Fe}$ & - & 3,73 & Décoction, 3 verres par jour pendant 3 jours. \\
\hline Pheonix dactylifera L. & Arecaceae & Fr & - & 13,37 & Décoction, 3 fois/jr pendant trois jours. \\
\hline Phyllantus amarus Shumach. & Phyllanthaceae & $\mathrm{Fe}$ & 1,17 & 0,78 & Décoction, tous les jours pendant une semaine. \\
\hline Piliostigma reticulatum (DC.) Hochst. & Caesalpiniaceae & $\mathrm{Fe}$ & 12,61 & 6,52 & Décoction, toute la journée pendant 3 jours. \\
\hline Pithecellobium dulce (Roxb.) Benth. & Mimosaceae & $\mathrm{Ec}$ & - & 23,48 & Décoction, 3 fois/jr pendant deux jours. \\
\hline Prosopis africana (Guill. et Perr.) Taub. & Mimosaceae & Ec & 7,70 & 6,67 & Décoction, voie orale 2 fois/jr. \\
\hline Sclerocarya birrea (A. Rich.) Hochst. & Anacardiaceae & $\mathrm{Fe}$ ou Ec & 82,71 & - & Décoction, 2 fois/jr pendant une journée \\
\hline Securinega virosa (Roxb. ex Willd.) & Euphorbiaceae & Rac & 10,01 & - & Décoction, 3 fois/jr pendant une semaine. \\
\hline Senna alata (L.) Roxb. & Caesalpiniaceae & $\mathrm{Fe}$ & 5,69 & - & Décoction, 2 fois/jr pendant deux jours. \\
\hline
\end{tabular}


D. NGUEMO DONGOCK et al. / Int. J. Biol. Chem. Sci. 12(1): 203-216, 2018

\begin{tabular}{|c|c|c|c|c|c|}
\hline Senna siamea Lam. & Fabaceae & $\mathrm{Fe}$ & - & 23,31 & Macération, toute une journée. \\
\hline Sorghum bicolor (L.) Moench. & Poaceae & $\mathrm{Gr}$ & - & 24,75 & Macération, toute une journée. \\
\hline Stereospermum kunthianum Cham. & Bignoniaceae & $\mathrm{Fe}$ & 10,53 & 5,50 & Décoction, tous les jours pendant 3 jours. \\
\hline Swartzia madagascariensis Desv. & Caesalpiniaceae & $\mathrm{Fe}$ & 8,29 & 11,08 & Décoction, 3 fois/jr pendant une semaine. \\
\hline Tamarindus indica L. & Caesalpiniaceae & $\mathrm{Fe}$ & - & 14,86 & Décoction, toute une journée. \\
\hline Tephrosia bracteolata Guill. \& Perr. & Fabaceae & $\mathrm{Fe}$ & 8,11 & 6,52 & Décoction, 1 verre 3 fois/jr pendant 3 jours. \\
\hline Terminalia avicennioides Guill.et Perr. & Combretaceae & $\mathrm{Fe}$ & 22,33 & 10,21 & Décoction, 1 verre trois/jr pendant trois jours. \\
\hline Zea mays L. & Poaceae & Brb & 22,84 & 14,00 & Décoction, 1 verre 3 fois /jr pendant une semaine. \\
\hline Ziziphus abyssinica Hochst. ex A. Rich. & Rhamnaceae & Rac & 16,10 & - & Décoction, 4 fois /jr pendant une semaine. \\
\hline
\end{tabular}

Tableau 2: Liste des éléments identifiés chez les espèces médicinales lors du screening phytochimique.

\begin{tabular}{lcccccc}
\hline & $\begin{array}{c}\text { Sclerocarya } \\
\text { birrea }\end{array}$ & $\begin{array}{c}\text { Detarium } \\
\text { microcarpum }\end{array}$ & $\begin{array}{c}\text { Annona } \\
\text { senegalensis }\end{array}$ & $\begin{array}{c}\text { Cassia } \\
\text { sieberiana }\end{array}$ & $\begin{array}{c}\text { Momordica } \\
\text { charantia }\end{array}$ & $\begin{array}{c}\text { Phylantus } \\
\text { amarus }\end{array}$ \\
\hline Tanins & +++ & +++ & +++ & +++ & +++ & +++ \\
Flavonoïdes & ++ & ++ & ++ & ++ & ++ & ++ \\
Alcaloïdes & +++ & +++ & +++ & +++ & ++++ \\
Saponosides & +++ & +++ & +++ & ++ & ++ & +++ \\
\hline \multicolumn{2}{r}{+++ Précipité abondant ;++ : Précipité moins abondant. } & &
\end{tabular}

+++ : Précipité abondant ; ++ : Précipité moins abondant. 


\section{DISCUSSION}

La forte proportion d'espèces végétales médicinales utilisées chez les Ngambaye pourrait s'expliquer, d'une part, par la richesse et la diversité de la flore de la région (Dongock et al., 2017), mais aussi par le fait que les Ngambaye sont originaires de la localité, les Laka et Kaba venant des environs, les Arabes étant des allogènes venus de la Région du Nord, par conséquent ne possèdent pas assez de connaissance sur cette végétation. Néanmoins, le nombre de plantes identifiées témoigne la bonne connaissance de la flore par ces groupes ethniques. Mapongmetsem (2005) dans ses travaux sur la représentation et gestion paysanne des jardins de case dans les hautes savanes guinéennes au Cameroun a également observé cette bonne maîtrise de la flore médicinale par la population concernée.

Les familles identifiées sont dominantes dans la flore de la région. Beunon (2011) à cet effet note que les Fabaceae Caesalpinoideae, Fabaceae Mimosoideae et Combretaceae sont les familles dominantes dans la zone de Pala au Tchad, ce qui indiquerait la valorisation de la flore par la population pour leurs divers besoins. Quelques espèces végétales identifiées dans la zone pour le traitement du diabète ont été également rapportées par d'autres auteurs: Persea americana au Cameroun (Nga et al., 2016); Catharanthus roseus et Senna siamea au Benin (Adomou et al., 2012).

Certaines espèces de la zone d'étude ont également fait l'objet de recherche par d'autres auteurs: Beunon (2011) au Tchad, Bene et al. (2016) et Yapi et al. (2015) en Côte D'Ivoire, Adomou et al. (2012) au Benin et Bayaga et al. (2017) à Akonolinga (Cameroun) où ils rapportent que les feuilles de Sclerocarya birrea, Momordica charantia et Moringa oleifera y sont les plus utilisées par les tradithérapeutes. Dongock et al. (2017) notent que les feuilles seraient fortement sollicitées par les traditherapeutes en raison de leurs richesses en substance actives. Les écorces occupent également une place de choix dans la pharmacopée tchadienne. Après les feuilles et les écorces, les racines occupent aussi une place importante dans la médecine traditionnelle. Ces faits confirment avec Houmenou et al. (2017) que la position souterraine des racines favoriserait la conservation des substances actives provenant des autres organes, les principes actifs étant des substances chimiques très labiles à la lumière; Il est prouvé que l'impact d'une utilisation particulière sur un arbre dépend de la partie utilisée et de la méthode de prélèvement. La récolte des racines d'un arbre et/ou de son écorce semble avoir plus d'incidences écologiques néfastes que la récolte des feuilles.

Le brassage des mœurs des populations entrâne parfois des changements dans la manière de faire et de vivre (Mapongmetsem, 2005). La décoction est le mode généralement le plus pratiqué en médecine traditionnelle, il a par ailleurs été noté par plusieurs auteurs avec, entre autres, Nga et al. (2016), Yapi et al. (2015), Yedomonhan et al. (2012), Béné et al (2016) et Diarra et al (2016). Les recettes des préparations sont monospécifiques ou en association de plusieurs espèces (plurispecifiques), ce qui permettrait d'après Bebbe (2006), de compléter et de renforcer l'efficacité du médicament.

L'abondance des précipités chimiques dans les différents organes des plantes médicinales utilisées témoignent ainsi leur utilisation par les différents groupes. Mariam (2006), Nga et al. (2016) et Yapi et al. (2015) rapportent aussi que les saponosides, les tanins notamment les tanins catéchiques, les stérols, terpènes, alcaloïdes, les flavonoïdes ont été présents dans les plantes utilisées dans le traitement traditionnel. Les tanins sont reconnus pour leur pouvoir de fixation aux protéines avec tendances à l'imperméabilité des couches sous-jacentes (Sambo, 2006). D'après Iserin (2001), les tanins sont des composants polyphénoliques qui contractent les tissus en liant les protéines et en les précipitant, permettant ainsi de stopper les hémorragies et de lutter contre les infections. De ce fait, il faut dire que les tanins contenus dans les échantillons pourraient prévenir et guérir les infections qui sont susceptibles de survenir chez le diabétique, justifiant l'utilisation traditionnelle de Sclerocarya birrea, Detarium microcarpum, Cassia sieberiana, Momordica charantia, Annona senegalensis et Phyllantus amarus. De nombreuses plantes médicinales qui sont 
traditionnellement utilisées dans le traitement du diabète contiennent les polyphénols qui seraient à l'origine de leurs effets thérapeutiques (Scalbert et al., 2005).

Sakine et al. (2012) et Gandonou et al. (2017) ont rapporté la présence des Tanins galliques, Tanins catéchiques, Alcaloïdes, Flavonoïdes, Stéroïdes, Terpènes, Leucoanthocyanes, Anthocyanes, Mucilages, Composés réducteurs et Coumarines dans les extraits de Boscia senegalensis, Colocynthis vulgaris et Lippia multiflora. Lippia multiflora est utilisée par les béninois comme plante hypertensive (Gandonou et al., 2017), Boscia senegalensis et Colocynthis vulgaris sont utilisées contre le diabete au Tchad (Sakine et al., 2012). C'est ainsi que Mathisen et al. (2002) ont également signalé dans les tissus des feuilles de Cassia sieberiana une abondance des dérivés flavonoïdes qui sont des $O$-flavonolosides parmi lesquels se trouvent d'importantes quantités de quercitrine et d'isoquercitrine pour la même plante (Maïga et al., 2005).

Il a été rapporté par Gandonou et al. (2017) que les tanins antibactérien possèdent des activités antivirales et antitumorale, sont anti-inflammatoires, anti-hypertenseurs, antimutagènes, immunostimulants, antidiarrhéique. Les coumarines présentent des propriétés antioxydants et antiinflammatoires. Les flavonoïdes présenteraient des activités antivirales, antitumorales, anti-inflammatoires, antihypertensives, antiallergiques et anticancéreuses.

Ces résultats différents un peu des nôtres qui signalent la présence en abondance de ces deux principes actifs dans les plantes. La richesse des plantes étudiées en saponoides pourraient aider à prévenir les complications dégénératives (cécité, neuropathie des jambes chez le diabétique (Adiza, 2007). Les saponosides sont connus pour leurs efficacités dans le traitement de nombreuses maladies. C'est ainsi que, Sereme et al. (2008) rapportent que dans les plantes, les saponosides sont des tensioactifs qui réguleraient l'hypertension artérielle. N'Guessan (2009) a également noté les précipités de stérols, polyterpènes, polyphénols, flavonoïdes, tanins catéchiques et alcaloïdes les feuilles de Phyllantus amarus. L'effet hypotenseur de cette plante est dû aux alcaloïdes et aux tanins se trouvant dans les feuilles (Bruneton, 1999). Cet auteur souligne ainsi que l'action des flavonoïdes sur les parois des vaisseaux sanguins aide à prévenir les troubles circulatoires, ce qui pourrait justifier cette utilisation traditionnelle des feuilles de Phyllantus amarus pour le traitement de l'hypertension artérielle. Les composés chimiques contenus dans les plantes, pourraient aussi justifier leur utilisation empirique dans diverses médecines traditionnelles.

\section{Conclusion}

Les populations tchadiennes ont de bonnes connaissances sur les maladies cardiovasculaires notamment le diabète et l'hypertension artérielle. L'enquête auprès de Ngambaye, Laka, Kaba et Arabes a permis de recenser 58 plantes médicinales reparties en 27 familles et 55 genres. Les Ceasalpiniaceae, Combretaceae et Euphorbiaceae sont majoritaires. Ces plantes sont valorisées en deux types de recettes qui sont monospécifiques ou faite à base de plusieurs plantes. Globalement, trois modes de préparations ont été enregistrés : la décoction (62\%), la macération $(23,66 \%)$ et l'infusion (14,34\%). Les feuilles $(27,32 \%)$, les écorces du tronc $(16,82 \%)$ et les racines $(14,76 \%)$ sont les principales parties utilisées. Les plantes très sollicitées dans le traitement des maladies cardiovasculaires soumises aux screening phytochimique ont relevé la présence abondante des tanins, des flavonoïdes, des alcaloïdes et des saponosides dans les différentes parties utilisées, ce sont ainsi des éléments chimiques ayant un rôle très positif pour le soulagement des maladies cardiovasculaires.

\section{CONFLIT D'INTERETS}

Les auteurs de cet article déclarent qu'il n'y a aucun conflit d'intérêts.

\section{CONTRIBUTIONS DES AUTEURS}

DND a contribué à la conception et à la structuration de l'étude, à la recherche documentaire et à la rédaction de l'article. LBA et ME ont contribué à la conception, à la structuration de l'étude, la collecte des 
données et aux analyses de laboratoire. MPM a contribué à la conception et à la structuration de l'étude, à l'analyse, à l'interprétation des données et à la finalisation de l'article.

\section{REMERCIEMENTS}

Nos remerciements vont vers les enquêtés de Moundou qui ont accepté de partager gracieusement leurs savoir-faire et les recettes de traitement à base de plantes contre les maladies cardiovasculaires. Nous louons ici leur franche et aimable collaboration.

\section{REFERENCES}

Adiza A. 2007. Etude d'une recette traditionnelle, des écorces de tronc de Sclerocarya birrea hosch et de Uapaca togoensis Pax utilisées dans le traitement du diabète. Thèse de pharmacie, Université de Bamako, Mali. 141p.

Adjanahoun E J, Ahyi A, Aké A L, Dan D L, Daouda H, Delmas M, Souzade S, Garba M, Guindo S, Koyong A, N'golo D, Raynal J L, Saadatou M. 1980. Médecine traditionnelle et pharmacopée : contribution aux études ethnobotaniques et floristiques au Niger, Act Paris, p. 250.

Adomou A C, Yedomonhan H, Djossa B, Legba S I, Oumorou M, Akoegninou A. 2012. Étude Ethnobotanique des plantes médicinales vendues dans le marché d'Abomey-Calavi au Bénin. Int. J. Biol. Chem. Sci., 6 (2): 745-772.

Atlas du Diabète de la FID. 2015. Septième édition. p. 144.

Bayaga HN, Guedje NM, Biye E H. 2017. Approche ethnobotanique et ethnopharmacologique des plantes utilisées dans le traitement traditionnel de l'ulcère de Buruli à Akonolinga (Cameroun). Int. J. Biol. Chem. Sci. 11(4): 1523-1541. DOI https://dx.doi.org/10.4314/ijbcs.v11i4.10

Bebbé F, 2006. Caractérisation des plantes médicinales utilisées dans le traitement de la stérilité humaine par les Dii, les Mboum et les Peuls de l'Adamaoua au Cameroun. Mémoire de maîtrise. Université de Ngaoundéré, Cameroun, p. 54.

Béné K, Camara D, Fofie N B Y, Kanga Y, Yapi A B, Yapo Y C, Ambe S A, Zirihi G N. 2016. Étude ethnobotanique des plantes médicinales utilisées dans le Département de Transua, District du Zanzan (Côte
d'Ivoire). Journal of Animal \&Plant Sciences, $\quad$ 27(2): 4230-4250. http://www.m.elewa.org/JAPS;

Beunon T. 2011. Inventaire des plantes médicinales dans quelques écosystèmes de la sous-préfecture de PALA au TCHAD, Mémoire de Master, Université de Dschang, Cameroun, p 111.

Bruneton J. 1999. Pharmacognosie, Phytochimie, Plantes Médicinales. Editions TEC \& DOC : Paris ; 783-785.

Diarra ML, Mariko M, Mbaye MS, Noba K. 2016. Plantes médicinales utilisées dans le traitement traditionnel du paludisme à Bamako (Mali). Int. J. Biol. Chem. Sci. 10(4): 1534-1541. DOI : http://dx.doi.org/10.4314/ijbcs.v10i4.7

Dongock Nguemo D, Mapongmetsem PM, Abdoulaye M, Noiha Noumi V. 2017. Ethnological studies on melliferous plants of the Soudano-Sahelian Zone of Chad. Journal of Medicinal Plants Studies, 5(3): 193-198.

Gandonou DC, Ahissou H, Tokoudagba J-M, Dansou C. 2017. Ethnobotanical, phytochemical and toxicity analysis of a Beninese antihypertensive plant: Lippia multiflora. Int. J. Biol. Chem. Sci., 11(4): 1816-1828. DOI : http://dx.doi.org/ 10.4314/ijbcs.v11i4.31.

George PR. 1999. Guide des plantes médicinales, Encyclopedie vie et santé. 44-51.

Houmenou V, Adjatin A, Tossou, MG, Yedomonhan H, Dansi A, Gbenou J, Akoegninou A. 2017. Etude ethnobotanique des plantes utilisées dans le traitement de la stérilité féminine dans les départements de l'Ouémé et du plateau au Sud Bénin. Int. J. Biol. Chem. Sci., 11(4): $\quad 1851-1871 . \quad$ DOI: http://dx.doi.org/10.4314/ijbcs.v11i4.34.

Idriss L. 2012. Diabète et Hypertension: Sanofi s'engage pour mieux contrôler son marché. Journal du Cameroun.com., 3 : 5.

Iserin P. 2001. Encyclopédie des Plantes Médicinales. LAROUSSE/VUEF, Paris, 400p.

Maïga A, Diallo D, Fané S, Sanogo R, Paulsen BS, Cissé B. 2005. A surveyof toxic plants on the market in the district of Bamako, Mali: traditional knowledge compared with a literature dearch of modern pharmacology and toxicology. 
Journal of Ethnopharmacology, 96: 183193.

Mapongmetsem PM. 2005. Représentation et gestion paysannes des jardins de case agroforestiers dans la zone périurbaine de Ngaoundéré (Adamaoua, Cameroun). Cameroon Journal of Ethnobotany, 1(1) : 92-102.

Mariam CT. 2006. étude de la phytochimie et des activités biologiques de quelques plantes utilisées dans le traitement traditionnel de la dysménorrhée au Mali, Thèse de Pharmacie, 1-175.

Nga EN, Pouka CK, Boumsong PCN, Dibong S D, Mpondo Mpondo E. 2016. Inventaire et caractérisation des plantes médicinales utilisées en thérapeutique dans le Département de la Sanaga Maritime: Ndom, Ngambe et Pouma. J. Appl. Biosc., 106: 10333-10352.

N'Guessan K, Beugré K, Guédé NZ, Dossahoua T, Laurent A. 2009. Screening phytochimique de quelques plantes médicinales ivoiriennes utilisées en pays Krobou (Agboville, Côte-d'Ivoire), Sciences \& Nature, 6(1): $1-15$.

Organisation mondiale de la santé. 2011. Gouvernance santé et population. Stratégie OMS de coopération avec les pays en développement, p. 5.

Proteus. 2000. Médecine alternative et plantes. Communiqué de presse organisation mondiale de santé. http:// www.reseauproteus.net/therapie/pharmach /-34k.

RGPH2. 2009. Deuxième Recensement General de la Population et de l'Habitat. Résultats globaux. Ministère de l'Economie et du plan. N'Djamena, Tchad. p. 83.

Sakine MNA, Mahmout Y, Gbenou J, Agbodjogbe W, Moudachirou M. 2012. Inventaire ethnobotanique des plantes du Tchad utilisées contre le diabète : effet anti-hyperglycémiant des extraits de Boscia senegalensis (Pers.) Lam. ex Poiret et de Colocynthis vulgaris (Schrad.). Revue CAMES - Série Pharm. Méd. Trad. Afr., 16 : 1-13.

Sambo MH. 2006. Etude du traitement traditionnel du diabète par une recette et les écorces de tronc de Manilkara multinervis Dub (Sapotaceae). Thèse de pharmacie, Université de Bamako, Mali. p. 41.

Scalbert A, Manach C, Morand C. 2005. Dietary polyphenols and the prevention of diseases. Crit Rev Food Sci Nutr., 45: 287 306.

Sereme A, Millogo-Rasolodimby J, Guinko S, Nacro M. 2008. Propriétés thérapeutiques des plantes a tanins du Burkina Faso, Pharmacopée et Médecine Traditionnelle Africaines, 15: 41-44.

Sharma P, Boyers D, Boachie C, Stewart F, Miedzybrodzka Z, Simpson W, Kilonzo M, Namee P M, Mowatt G. 2012. Elucigene FH20 and LIPOchip for the diagnosis of familial hypercholesterolaemia: a systematic review and economic evaluation, Health Technology Assessment NIHR HTA programm www.hta.ac.uk. (Executive summary) 16(17): $\quad$ DOI: 10.3310/hta16170.

Sougnabé P. 2010. Pastoralisme en quête d'espace en savane tchadienne : des Peuls autour de la forêt classée de Yamba Berté. Thèse de Doctorat, EHESS-Paris, France, p. 419.

Tearfund B, Isabel C, Bridgnarth S. 2001. Bulletin d'informations trimestriel pour lier les agents de développement du monde entier: Pas à Pas, les médecines traditionnelles. 1- 48.

Ueli Z, Lic P, Bopp M. 2008. Chiffres et données sur les maladies cardiovasculaires en Suisse. Fondation Suisse de Cardiologie; 1-47.

Yedomonhan H, Houenon GJ, Akoegninou A, Adamou AC, Tossou MG, Maesen LJ. 2012. The woody flora and its importance for honey production in Sudano-Guinean zone in Benin. International Journal of Science and Advanced Technology, 2(3): 64-72.

Yapi AB, Kassi NJ, Fofie NBY, Zirihi GN. 2015. Etude ethnobotanique des Astéracées médicinales vendues sur les marches du district autonome d'Abidjan (Cotes d'Ivoire). Int. J. Biol. Chem. Sci., 9(6): 2633-2647. 